\title{
Anillos de crecimiento de Pinus hartwegii como indicadores de fluctuaciones climáticas y de la influencia de fenómenos océano-atmósfera en la Faja Volcánica Transmexicana
}

\author{
Tree-rings of Pinus hartwegii as indicators of climatic fluctuations and the influence \\ of ocean-atmosphere phenomena in the Transmexican Volcanic Belt
}

\author{
Ulises Manzanilla-Quiñones', Oscar Alberto Aguirre-Calderón², José Villanueva-Díaz, \\ Aldo Rafael Martínez-Sifuentes ${ }^{3}$ y Patricia Delgado-Valerio'
}

\begin{abstract}
l Universidad Michoacana de San Nicolás de Hidalgo. Facultad de Agrobiología "Presidente Juárez". Uruapan, Michoacán, México.

2 Universidad Autónoma de Nuevo León. Facultad de Ciencias Forestales. Linares, Nuevo León, México.
\end{abstract}

\author{
3 Instituto Nacional de Investigaciones Forestales _ * * Autor de correspondencia. jvilladi@prodigy.net.mx \\ Agrícolas y Pecuarias. Centro Nacional de \\ Investigación Disciplinaria en Relación Agua, Suelo, \\ Planta, Atmósfera. Gómez Palacio, Durango, México.
}

\section{RESUMEN}

El presente estudio evalúa la respuesta climática y la influencia de fenómenos océano-atmósfera sobre el crecimiento radial de Pinus hartwegii Lindl en el Nevado de Colima (NEC), Nevado de Toluca (NET) y Pico de Orizaba (POR). Se fecharon 78 núcleos de crecimiento, a los cuales se les midió el ancho de anillo total. La calidad del fechado y generación de cronologías se realizaron en los programas dendrocronológicos. Los análisis de respuesta entre series dendrocronológicas y variables climáticas e índices del Niño Oscilación del Sur (ENOS), Oscilación Decadal del Pacífico (ODP) y Oscilación Multidecadal del Atlántico (OMA) se realizaron a través de correlaciones Bootstrap. Mediante análisis de regresión se generaron, calibraron y verificaron modelos lineales, con los cuales se logró reconstruir 57\% y 53\% de la variabilidad instrumental de la precipitación de noviembre-mayo para NEC (1940-2016) y diciembre-mayo para NET (1928-2016). Los años de sequías intensas de 1930, 1949, 1955, 1959, 1960, 1991, 1998 y 1999 y de humedad extrema de 1992, coinciden con las reconstrucciones de precipitación para NEC y NET. La temperatura máxima tuvo un efecto negativo significativo en el crecimiento radial de $P$. hartwegii de los tres sitios. La influencia de ENOS sobre el crecimiento arbóreo fue significativo $(\mathrm{p}<0.05)$ solamente en NEC. La influencia de ODP y OMA fue significativo en los tres sitios $(\mathrm{p}<0.05)$. Las condiciones microambientales locales donde se desarrolla $P$. hartwegii en los tres sitios de estudio modifican la respuesta fisiológica al clima y modulan el efecto de los fenómenos océano-atmosféricos.

PALABRAS CLAVE: bosque de alta montaña, dendrocronología, índice de ancho de anillo, precipitación reconstruida, series de crecimiento, variabilidad climática.

\section{ABSTRACT}

The present study evaluates the climatic response and the influence of ocean-atmosphere phenomena on the radial growth of Pinus bartwegii Lindl in Nevado de Colima (NEC), Nevado de Toluca (NET) and Pico de Orizaba (POR). Seventy-eight growth cores were dated, and their total ring width was measured. Dating quality and chronology generation were performed in dendrochronological programs. Response analyses between dendrochronological series and climatic variables and the Niño Southern Oscillation (ENSO), Pacific Decadal Oscillation (PDO) and Atlantic Multidecadal Oscillation (AMO) indices were performed through Bootstrap correlations. Linear models were generated, calibrated, and verified through regression analysis, with which $57 \%$ and $53 \%$ of the instrumental variability of November-May precipitation for NEC (1940-2016) and December-May for NET (1928-2016) were reconstructed. The intense drought years of 1930,1949, 1955, 1959, 1960, 1991, 1998, and 1999 and extreme wetness of 1992 coincided with the precipitation reconstructions for NEC and NET. Maximum temperature had a significant negative effect on radial growth of $P$. hartwegii at the three sites. The influence of ENSO on tree growth was significant $(\mathrm{p}<0.05)$ only at NEC. The influence of ODP and OMA was significant at all three sites $(\mathrm{p}<0.05)$. The local microenvironmental conditions where $P$. hartwegii develops in the three study sites modify the physiological response to climate and modulate the effect of ocean-atmospheric phenomena.

KEYWORDS: high mountain forest, dendrochronology, ring width index, reconstructed precipitation, growth series, climatic variability. 


\section{INTRODUCCIÓN}

Las cronologías generadas a partir de la estandarización de mediciones del grosor de anillos de crecimiento de especies arbóreas han aportado datos relevantes sobre las fluctuaciones interanuales $\mathrm{y} / \mathrm{o}$ multianuales del comportamiento climático, la influencia de fenómenos océano-atmósfera como El Niño Oscilación del Sur (ENOS), la Oscilación Decadal del Pacífico (ODP) y la Oscilación Multidecadal del Atlántico (OMA) (Biondi, Gershunov y Cayan, 2001; Villanueva-Díaz et al., 2015, Stahle et al., 2016).

Asimismo, han ayudado en la reconstrucción de eventos ambientales como sequías extremas y permitido determinar la frecuencia e intensidad de incendios forestales (Villanueva-Díaz et al., 2015; Aquino-Ramírez et al., 2019; Cerano-Paredes et al., 2019; Carlón, Villanueva, Soto, Mendoza y Macías, 2021; Martínez-Sifuentes, Villanueva-Díaz y Estrada-Ávalos, 2020). Así como en la generación de índices como el de severidad de sequías de Palmer (PDSI) (Cook, Seager, Cane y Stahle, 2007; Stahle et al., 2016) y el índice normalizado de precipitación (SPI) (Stahle et al., 2020).

La investigación dendrocronológica ha aumentado significativamente en las dos últimas décadas en México, debido a que los anillos de crecimiento son excelentes bioindicadores de alta resolución y a una escala anual y estacional fina, de los cambios ecológicos y climáticos presentes del sitio (Fritts, 1976; Schofield et al., 2016; Villanueva-Díaz et al., 2018a). Por ello, las aplicaciones de la dendrocronología han permitido a los investigadores evaluar eventos y determinar su influencia en el crecimiento arbóreo (Villanueva-Díaz et al., 2015; Stahle et al., 2016; Carlón et al., 2021).

En el caso específico de los bosques templados de coníferas de México, diferentes estudios han identificado a la precipitación como el principal factor limitante en el crecimiento arbóreo (Villanueva-Díaz et al., 2015; Gutiérrez-García y Ricker 2019; Carlón et al., 2021), siendo la mayoría de estos, generados a partir de taxa pertenecientes a los géneros Pinus, Pseudotsuga, Abies,
Taxodium y en menor proporción a Quercus y Juniperus (Villanueva-Díaz et al., 2015; Acosta-Hernández, PompaGarcía y Camarero, 2017).

Los ecosistemas forestales de alta montaña en México son considerados como zonas sensibles a los cambios en temperatura y precipitación (Villanueva-Díaz et al., 2015; 2016, Astudillo-Sánchez, Fowler, Villanueva-Díaz, EndaraAgramont y Soria-Díaz, 2019), ideales para analizar y examinar la respuesta climática de las especies alpinas arbóreas al calentamiento global actual, el cual se prevé que afectaría severamente a múltiples ecosistemas a futuro cercano (Sáenz-Romero et al., 2010; Panel Intergubernamental de Expertos sobre Cambio Climático [IPCC], 2014; Manzanilla-Quiñones, Aguirre-Calderón, Jiménez-Pérez. Treviño-Garza y Yerena-Yamallel, 2019).

En estos ecosistemas de alta montaña el principal factor estresante de crecimiento en los árboles es la variabilidad de la temperatura durante el período vegetativo de primavera, cuando se reactiva la actividad celular del cambium vascular arbóreo (Villanueva-Díaz et al., 2016; Gutiérrez-García y Ricker, 2019).

A su vez, el interés por estudiar los ecosistemas alpinos ha aumentado en las últimas décadas (Körner, 1998; Villanueva-Díaz et al., 2016; Astudillo-Sánchez et al., 2019), con el fin de analizar el efecto del calentamiento global en estos ecosistemas, que se infiere, obligará a ciertas especies a desplazarse altitudinalmente para subsistir a las nuevas condiciones climáticas (Sáenz-Romero et al., 2010; Kohler et al., 2014; Manzanilla-Quiñones et al., 2019). Actualmente, este efecto se refleja en un aumento significativo en el crecimiento radial de especies alpinas como Juniperus monticola Martínez y Pinus hartwegii Lindl, derivado de estaciones más cálidas y prolongadas (Villanueva-Díaz et al., 2016; Astudillo-Sánchez et al., 2019).

$P$. hartwegii es una especie de pino de alta montaña que se distribuye desde el norte de México hasta las partes altas de Guatemala y Honduras. La especie posee un tronco recto, de corteza rugosa y escamosa de color gris, fascículos de tres a cinco acículas, de hasta $100 \mathrm{~cm}$ de diámetro y una altura de 25 m a 30 m (Farjon, Pérez de la Rosa y Styles, 1997). 
Los ejemplares de $P$. hartwegii suelen alcanzar edades superiores a los 400 años (Villanueva-Díaz et al., 2015) y producen anillos anuales de crecimiento bien definidos (madera temprana y madera tardía bien diferenciadas) con un porcentaje muy bajo $(0.31 \%)$ de presencia de anillos ausentes y de anillos falsos $(5 \%<$ ) (Gutiérrez-García y Ricker 2019; Manzanilla-Quiñones, Aguirre-Calderón, Jiménez-Pérez y Villanueva-Díaz, 2020).

La línea alpina de $P$. hartwegii en México se encuentra distribuida en un gradiente altitudinal de $2800 \mathrm{~m}$ a $4300 \mathrm{~m}$ s.n.m. (Farjon, Pérez de la Rosa y Styles, 1997), donde a partir de los $3000 \mathrm{~m}$ forma bosques puros, llamados bosques subalpinos (Manzanilla-Quiñones et al., 2019). Dado que los árboles de las líneas alpinas integran en sus anillos de crecimiento las respuestas fisiológicas de los cambios anuales en el ambiente (Fritts, 1976; VillanuevaDíaz et al., 2016; Astudillo-Sánchez et al., 2019), estas fluctuaciones constituyen una valiosa base de información relevante sobre la variación climática, la cual se puede extraer mediante la aplicación de técnicas dendrocronológicas.

Aunado a lo anterior, el estudio de los fenómenos océano-atmósfera como ENOS, ODP y OMA y su impacto en el crecimiento de las especies de alta montaña ha sido poco estudiado en México (Villanueva-Díaz et al., 2016; Astudillo-Sánchez, Villanueva-Díaz, EndaraAgramont, Nava-Bernal, Gómez-Albores, 2017).

Estudios recientes realizados en $P$. hartwegii de las partes altas de las montañas del centro de México mencionan una influencia positiva y significativa de ODP en el crecimiento radial de la especie, así como una muy baja de influencia de ENOS y OMA en el crecimiento radial de P. hartwegii de la zona (Manzanilla-Quiñones et al., 2020).

Bajo esta premisa, se planteó la siguiente pregunta de investigación ¿la influencia climática y atmosférica (ENOS, ODP y OMA) en $P$. hartwegii será igual en sitios con condiciones ambientales similares de alta montaña dentro de la Faja Volcánica Transmexicana (FVTM)?

\section{OBJETIVOS}

Para contestar a la interrogantes planteada, se formularon los siguientes objetivos específicos i) identificar las variables climáticas que más influyen en el crecimiento radial anual de $P$. hartwegii en tres sitios de alta montaña dentro de la FVTM, ii) reconstruir la variable climática más relevante del período de función de respuesta de cada sitio y iii) evaluar la influencia de ENOS, ODP y OMA en el crecimiento radial de los árboles muestreados.

\section{MATERIALES Y MÉTODOS}

\section{Sitios de estudio}

Los sitios de estudio están situados dentro de las áreas naturales protegidas Nevado de Colima (NEC), Nevado de Toluca (NET) y Pico de Orizaba (POR) en la FVTM (Fig. 1). Las coordenadas geográficas de los sitios son $19^{\circ} 35^{\text {‘ }}$ 2.5” latitud norte y $103^{\circ} 35^{\prime} 44.8^{\prime}$ " longitud oeste (NEC), $19^{\circ} 7$ ' 13.1" latitud norte y $99^{\circ} 48^{\prime} 57.7^{\prime \prime}$ longitud oeste (NET) y $19^{\circ} 1$ ' 26.1" latitud norte y $97^{\circ} 20^{\prime} 17.6^{\prime}$ ' longitud oeste (POR).

Los árboles muestreados en los sitios de estudio se caracterizan por crecer en un clima $\mathrm{Cb}^{\prime}(\mathrm{w} 2)$ (semifrío, subhúmedo), con una temperatura media anual que oscila entre $5{ }^{\circ} \mathrm{C}$ y $12{ }^{\circ} \mathrm{C}$ y una estación de lluvias de verano (García, 1998). La altitud promedio de los sitios estudiados fue de $3404 \mathrm{~m}$ (NEC), $3851 \mathrm{~m}$ (NET) y $4023.8 \mathrm{~m}$ (POR). La exposición fue suroeste (NEC), sureste (NET) y este (POR). La pendiente fue de 2.5\% (NEC), 3.1\% (NET) y $3.4 \%$ (POR). Los suelos dominantes corresponden a los tipos Andosol mólico (Tm) para NEC, Andosol húmico (Th) para NET y Andosol ócrico (To) para POR (Instituto Nacional de Estadística y Geografía [Inegi], 2014). De acuerdo con Inegi (2016) el uso del suelo y vegetación registrados en los sitios de estudio corresponde bosque de oyamel (Abies religiosa) para el NEC, bosque de pino (Pinus hartwegii) para el NET y pradera alpina para el POR. 


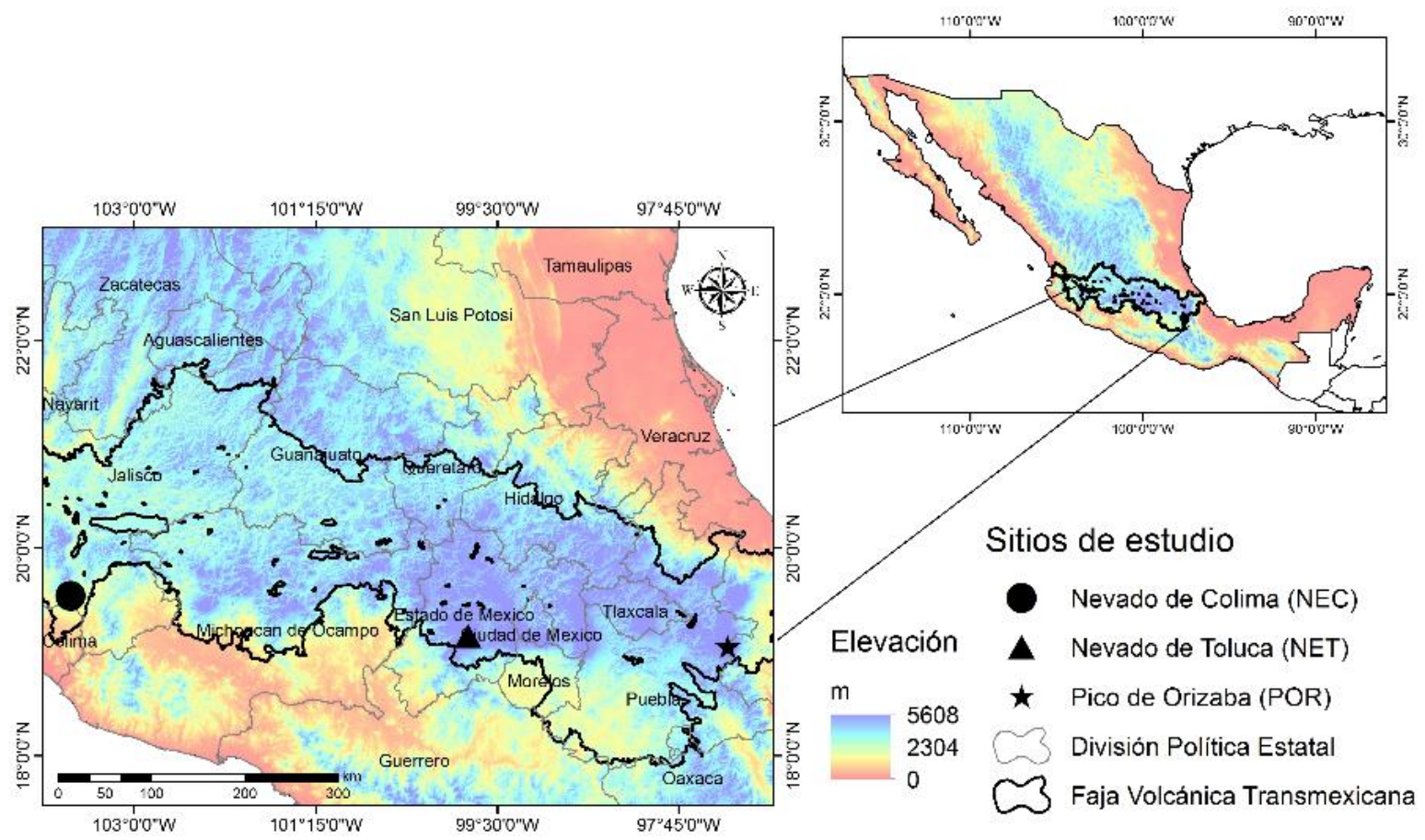

FIGURA 1. Localización geográfica de los sitios de estudio.

\section{Muestreo}

El muestreo dendrocronológico se realizó en tres sitios de alta montaña, durante los meses de febrero a marzo de 2017. En cada sitio, se seleccionaron 18 árboles en NET, 18 en POR y 20 en NEC de P. hartwegii, se evitó muestrear ejemplares dañados (deformados, quemados o con signos de presencia de plagas y/o enfermedades). Los árboles muestreados presentaron diámetros promedio de $79.2 \mathrm{~cm}$ (NEC), $55.6 \mathrm{~cm}$ (NET) y $37.9 \mathrm{~cm}$ (POR), respectivamente. A cada espécimen muestreado, se les extrajo dos núcleos de incremento mediante el uso de un taladro de Pressler marca Haglöf de $50 \mathrm{~cm}$ de longitud y un diámetro de $5.1 \mathrm{~mm}$. Las muestras se obtuvieron a una altura de $1.3 \mathrm{~m}$ del suelo y en dirección perpendicular a la pendiente, con el fin de evitar madera de tensión y compresión; posteriormente las virutas fueron colocadas en popotes de plástico, previamente perforados para su transporte al laboratorio.

\section{Generación de series de crecimiento}

Las muestras se montaron en moldes de madera, para después ser pulidas con lijas de diferentes granos $(60,80$, $120,280,600$ y 1200), para posteriormente ser prefechadas y analizadas mediante técnicas dendrocronológicas. La medición del ancho total de anillo se realizó en un equipo VELMEX ${ }^{\circledR}$ (precisión $0.001 \mathrm{~mm}$ ) en el Laboratorio Nacional de Dendrocronología en Gómez Palacio, Durango, México. La calidad de la datación de los anillos de crecimiento se realizó en el programa Cofecha, a través de correlaciones de Pearson entre las muestras estandarizadas de ancho de anillo en segmentos de 50 años traslapados cada 25 años. Para que el fechado sea estadísticamente fiable, este debe tener un valor de intercorrelación entre series mayor a 0.328 ( $\mathrm{p}<0.01$ ) (Holmes, 1983). Por lo tanto, series de crecimiento con valores de intercorrelación $<0.328(\mathrm{p}>0.05)$ fueron descartadas del anáisis. 
Las cronologías se generaron en el programa Arstan, el cual estandarizó las series de crecimiento de ancho de anillo (Cook y Holmes, 1986). Este procedimiento, se llevó a cabo al aplicar una función exponencial negativa, y luego un Spline cubico suavizado de 128 años, conservando 50\% de la varianza y $67 \%$ de la longitud de la serie, lo cual permitió eliminar la máxima variabilidad no atribuida al clima (Pompa-García, Dávalos-Sotelo, Rodríguez-Téllez, Aguirre-Calderón y Treviño-Garza, 2014). La estandarización de las series individuales con media igual a 1.0 y varianza homogénea, se obtuvo al dividir las mediciones de ancho de anillo de cada año entre el valor de la curva de ajuste previamente establecida (Cook, 1987). La aplicación de dichos comandos permitió eliminar los factores externos (ruido) ajenos a la señal climática de interés (Cook, 1987), tendencias biológicas del crecimiento y a maximizar las variaciones climáticas de alta frecuencia (interanual) registradas en los anillos de crecimiento (Fritts, 1976; Cook, 1987). Arstan generó tres cronologías: estándar, residual y arstan, con las cuales se realizaron análisis de correlación Bootstrap entre los datos climáticos con la finalidad de encontrar períodos de función de respuesta significativos en el crecimiento anual radial de $P$. hartwegii.

\section{Parámetros estadísticos de cronología}

Existen diversos parámetros estadísticos empleados para evaluar la calidad de una cronología, como la correlación entre series (correlación de Pearson entre muestras), sensibilidad media (cambio relativo en el grosor de un anillo de crecimiento de un año previo al año actual de crecimiento), autocorrelación de primer orden (porcentaje de influencia del año anterior en el crecimiento anual actual), variación del primer componente principal (porcentaje de variabilidad común entre los árboles de una serie de crecimiento) y la señal expresada de la población (SEP) utilizada para cuantificar la señal común de crecimiento de una población hipotética con un tamaño de muestra infinito, cuyo valor para ser estadísticamente confiable debe ser > 0.85 (Fritts, 1976; Briffa y Jones, 1990; Speer, 2010).

\section{Datos climáticos}

La información climática disponible se descargó de la plataforma Clicom (Centro de Investigación Científica y de Educación Superior de Ensenada [Cicese], s/f) para las estaciones meteorológicas con registros $>30$ años y cercanas a los sitios de estudio.

Para el sitio NEC se obtuvieron los datos de 5 estaciones meteorológicas (Comala, Cuauhtémoc, Quito, San Marcos y el Nogal), 11 estaciones (Mexicalcingo, Calixtlahuaca, Coatepec Harinas, El Islote, Nevado de Toluca, San Francisco Oxtotilpan, Santa María del Monte, Tenango, Toluca DGE, Toluca OBS y Toma Tecomatepec) para NET y seis estaciones (Santa Cruz Coyotepec, Cuesta Blanca, Ciudad Serdán, Guadalupe Victoria, Palmar de Bravo y San Pedro Temamantla) para POR. Sin embargo, los registros meteorológicos presentaron datos incompletos, por lo que fue necesario estimar lo datos faltantes mediante la identificación de períodos de actividad climática común, los cuales presentaron más de $85 \%$ de los registros completos.

Los datos faltantes de precipitación se estimaron mediante un análisis de regresión lineal simple entre estaciones meteorológicas; mientras que, los de temperatura se calcularon por medio de medias móviles orden $n=5$ (Schulz, 1976; Campos-Aranda, 1998).

En la figura 2 se presenta el comportamiento climático histórico de la precipitación y temperatura promedio mensual regional (promedio) de las estaciones meteorológicas cercanas a los sitios de estudio.

\section{Períodos de función de respuesta}

Las relaciones entre el clima y la cronología de cada sitio fueron determinadas mediante correlaciones Bootstrap en el programa Dendroclim (Biondi y Waikul, 2004). Los coeficientes de correlación mensual y estacional fueron obtenidos en una ventana temporal de enero del año de previo al crecimiento hasta diciembre del año actual de crecimiento (24 meses), esto debido a que estudios previos en la región de estudio mencionan que las condiciones climáticas anteriores al año de crecimiento de la especie 
influyen en el crecimiento anual actual (Biondi, Hartsough y Galindo-Estrada, 2005). Este análisis permitió identificar los meses individuales y/o estacionales que más influyeron en el crecimiento arbóreo de cada sitio.

\section{Reconstrucción de precipitación estacional}

Con base en los resultados obtenidos de los análisis de función de respuesta climática, se determinaron períodos significativos (que explicaran $>50 \%$ del comportamiento de la variable de interés) de respuesta, entre el clima y el crecimiento radial anual de $P$. hartwegii en cada sitio de estudio.

Los períodos totales de función de respuesta de los datos de precipitación de cada sitio de estudio fueron particionados en dos subperíodos; calibración y verificación. Donde los subperíodos deben ser significativos para poder generar la ecuación de reconstrucción de lluvia estacional acumulada.

La verificación de los modelos lineales de reconstrucción se respaldó en diversas pruebas estadísticas como la correlación de Pearson, reducción del error, valor de " $t$ " y primera diferencia significativa, análisis realizados en la subrutina "Verify" (Holmes, 1994) de la Librería de Programas Dendrocronológicos de la Universidad de Arizona DPL (Fritts, 1976). La autocorrelación de primer orden de residuales de los modelos de reconstrucción fue calculada mediante la aplicación de la prueba de DurbinWatson en el programa R (Core Team, 2019).

Los cambios contrastantes en los períodos de reconstrucción de la precipitación, como los años de sequía y humedad extrema, se identificaron como aquellos años con valores mayores a \pm 2 desviación estándar $(\sigma)$, en tanto que, dos o más años consecutivos por debajo o arriba de la media, se consideraron como períodos de secas o años anormalmente húmedos (Sun et al., 2018). Los resultados de los años de sequía y de humedad de cada sitio fueron comparados con los datos de severidad de sequias (PDSI) generados para México por el Atlas Mexicano de Sequías (Stahle et al., 2016).
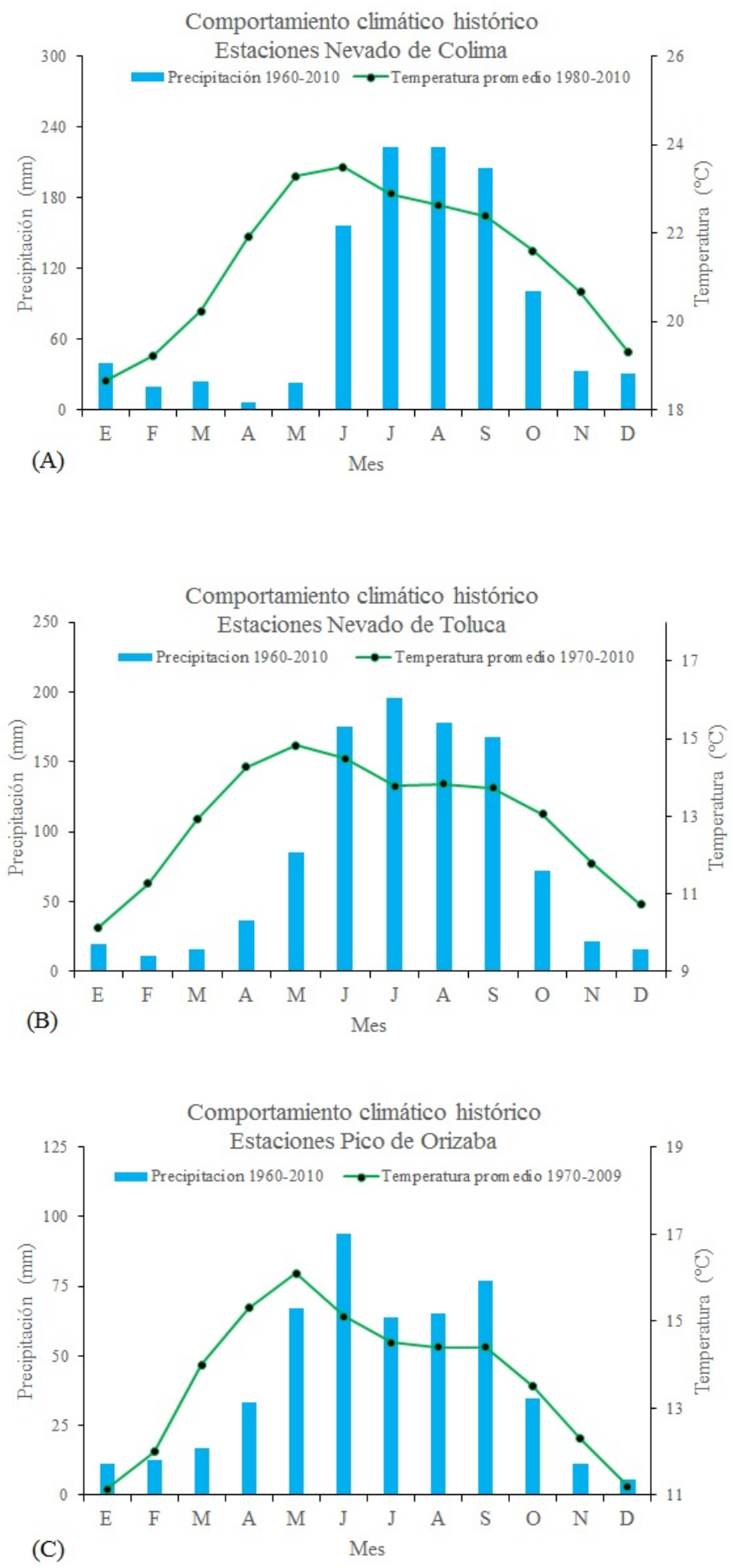

FIGURA 2. Tendencias de la precipitación y la temperatura promedio mensual históricas de las estaciones meteorológicas cercanas al Nevado de Colima (A), Nevado de Toluca (B) y Pico de Orizaba $(\mathrm{C})$ de la Faja Volcánica Transmexicana. 


\section{Influencia de fenómenos océano-atmósfera}

La influencia de los fenómenos océano-atmósfera sobre el crecimiento radial anual de $P$. hartwegii, se analizó a través de correlaciones Bootstrap entre los índices ENOS IEM (índice ENSO Multivariado), INO (índice Niño oceánico), IOS (índice oscilación del Sur) (NOAA Physical Sciences Laboratory [PSL], s/f a), ODP y OMA (PSL, s/f b) y las cronologías (estándar, residual y arstan) en el programa DendroClim (Biondi y Waikul, 2004) en una ventana temporal de 24 meses, de enero del año de previo a diciembre del año actual de crecimiento arbóreo.

\section{Resultados}

\section{Análisis dendrocronológico}

El número de muestras fechadas fue de 27 para NEC, 26 para NET y 25 para POR. El resto de las muestras se omitieron del análisis, porque no superaron el valor mínimo de correlación $(\mathrm{r}<0.328, \mathrm{p}<0.01)$ idóneo (Holmes, 1983). Los resultados de Cofecha indicaron valores de intercorrelaciones entre series de 0.585 (NEC), 0.516 (NET) y 0.412 (POR). La versión estándar de las cronologías indicó una sensibilidad media de 0.314 (NEC), 0.243 (NET) y 0.234 (POR). La autocorrelación de primer orden de la cronología fue de 0.244 (NEC), 0.621 (NET) y 0.338 (POR), lo que indica que las condiciones de crecimiento del año anterior muestran influencia en el crecimiento actual. La variación del primer componente principal (Eigen valor) fue de 10.19 (NEC), 7.53 (NET) y 4.08 (POR), lo que implica una varianza total de $40.33 \%, 37.64 \%$ y $21.48 \%$, respectivamente. Valores que representan la señal de crecimiento común entre los árboles muestreados. El tamaño de muestra adecuado para la SEP $(\geq 0.85)$ fue de nueve muestras (1940-2016) en NEC, 12 muestras (19282016) en NET y 18 muestras ( $\mathrm{SEP}=0.80,1960-2016)$ en POR (Fig. 3). Este último sitio, presentó los árboles más jóvenes de las tres áreas estudiadas.

\section{Respuesta climática}

El análisis de correlación de Pearson indicó una asociación positiva y significativa entre la cronología residual y la precipitación mensual de noviembre del año previo a mayo del año actual en NEC ( $\mathrm{r}=0.76, \mathrm{p}<0.01,1960-2006$, estación El Nogal) y de diciembre del año previo a mayo del año actual para NET $(r=0.74, p<0.01,1962-2009$, estación Mexicalcingo) y de diciembre del año previo a mayo del año actual en POR (estación Santa Cruz Coyotepec, 1974-2007, $\mathrm{r}=0.53, \mathrm{p}<0.01)$.

La temperatura máxima promedio mostró un efecto negativo en el crecimiento de la especies en los sitios estudiados; así la temperatura máxima promedio estacional de marzo a mayo indicó una asociación negativa con la cronología residual del NEC ( $r=-0.42, \mathrm{p}<0.05)$; en NET, la asociación con el período estacional de septiembre del año previo a febrero del año actual de crecimiento fue de $0.42(\mathrm{p}<0.05)$ y en POR de $-0.54(\mathrm{p}<0.01)$ para el período estacional de junio a octubre. La temperatura promedio mostró un efecto negativo durante mayo en NEC ( $\mathrm{r}=$ $0.38, \mathrm{p}<0.05)$ y de junio a diciembre en POR $(\mathrm{r}=-0.45, \mathrm{p}$ $<0.05)$, sin embargo, esta variable no tuvo efecto significativo ( $p>0.05)$ en el crecimiento radial de P. hartwegii en NET.

El crecimiento radial en los sitios NEC y NET no presentó una respuesta significativa con la temperatura mínima ( $p>0.05)$, en cambio, el sitio POR mostró una respuesta negativa para los meses de julio-octubre $(r=-0.53$, $\mathrm{p}<0.01)$. La figura 4 muestra el análisis de función de respuesta para una ventana temporal de 24 meses entre el clima (precipitación y temperatura máxima, promedio y mínima) y las cronologías (residual) para los tres sitios en estudio.

\section{Reconstrucción de precipitación}

De acuerdo con el análisis de la relación clima-crecimiento radial, se reconstruyó la precipitación estacional de noviembre del año previo a mayo del año actual en el sitio NEC y la precipitación de diciembre del año previo a mayo del año actual en el sitio NET, mediante la aplicación de modelos de regresión lineal. 

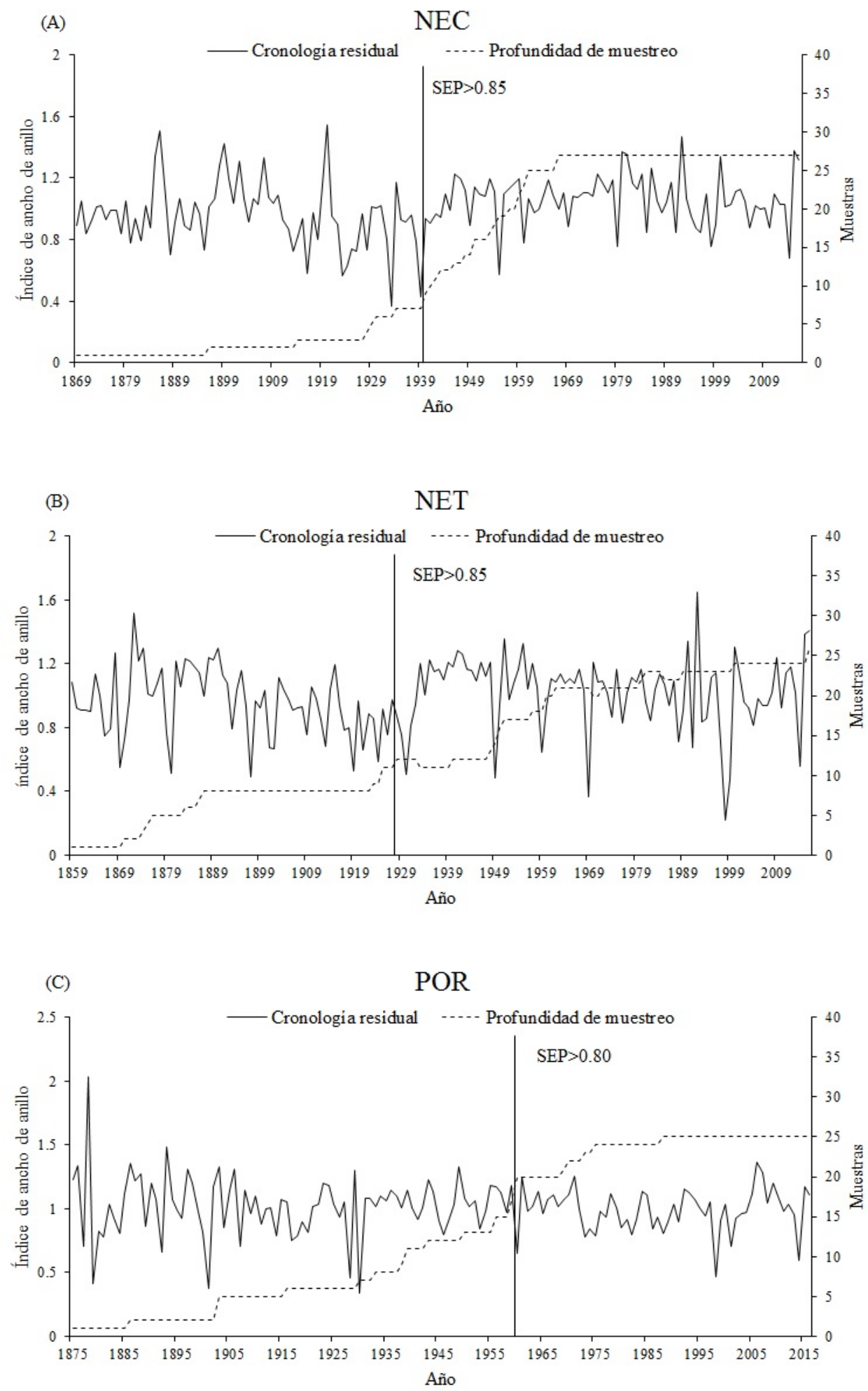

FIgURA 3. Profundidad de muestreo y valores SEP de las cronologías residuales de los sitios NEC (A), NET (B) y POR (C) de la Faja Volcánica Transmexicana. 

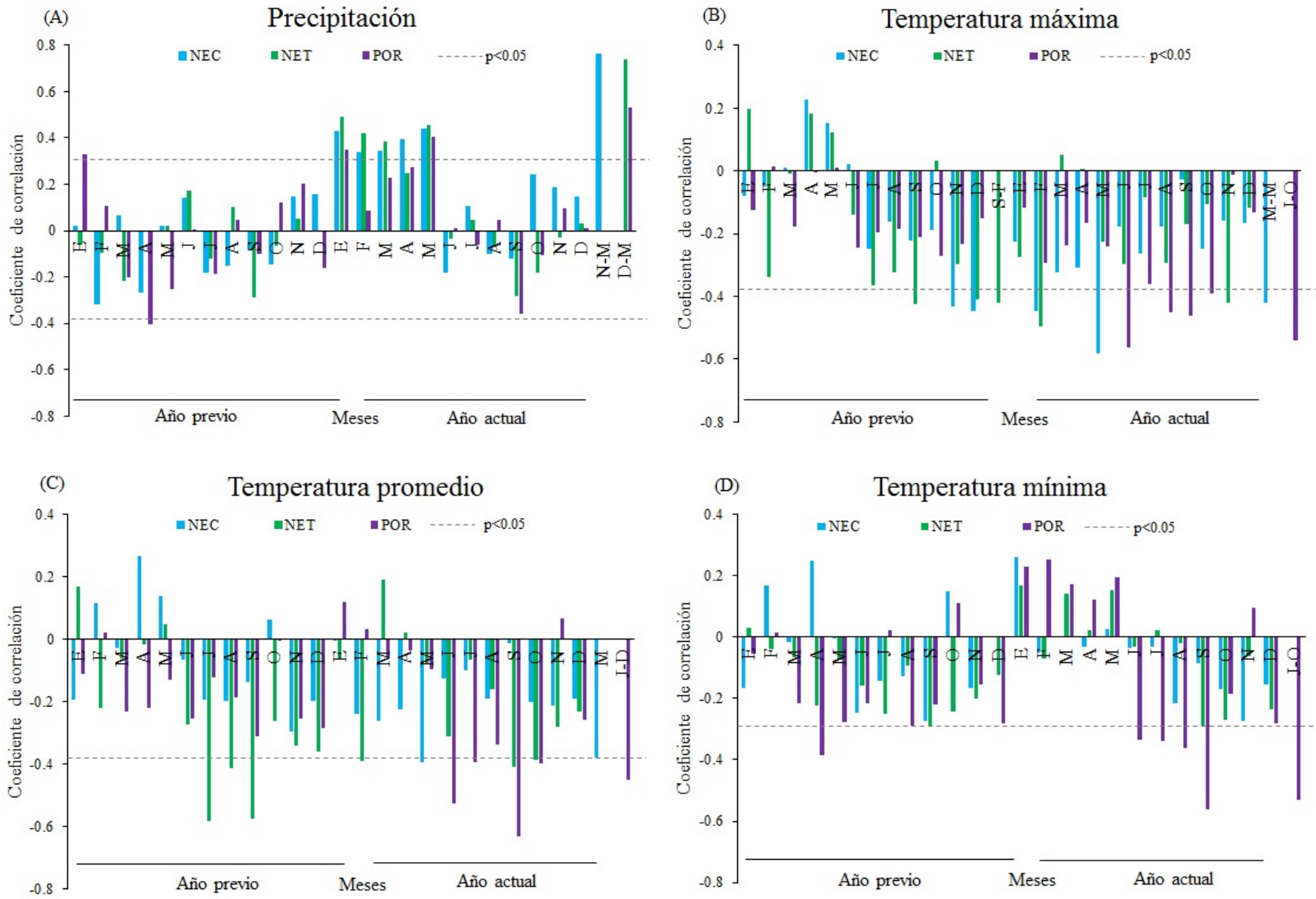

FIgura 4. Coeficientes de correlación de la cronología residual y los registros climáticos en los sitios NEC (Nevado de Colima), NET (Nevado de Toluca) y POR (Pico de Orizaba) de la Faja Volcánica Transmexicana.

Los valores superiores a las líneas punteadas horizontales son significativos (95\%).

Los períodos completos de los registros de precipitación para NEC (1960-2006) y NET (1962-2009) se utilizaron para generar a través de análisis de regresión los modelos lineales. El análisis de calibración de los modelos de reconstrucción comprendió dos subperíodos 1960-1983 ( $\mathrm{r}^{2}$ $=0.38, \mathrm{p}<0.01)$ y $1984-2006\left(\mathrm{r}^{2}=0.73, \mathrm{p}<0.01\right)$ para NEC y de $1962-1985\left(\mathrm{r}^{2}=0.12, \mathrm{p}>0.01\right)$ y $1986-2009\left(\mathrm{r}^{2}\right.$ $=0.70, \mathrm{p}<0.01)$ para NET (Tabla 1; Fig. 5). La verificación se realizó con los subperíodos no utilizados en la calibración y viceversa.

Los resultados de las pruebas de calibración y verificación indicaron que ambos períodos pasaron las pruebas estadísticas por lo que se consideraron estadísticamente robustos para fines de reconstrucción en los dos sitios (Tabla 2). Los valores del estadístico DurbinWatson fueron de 1.95 (NEC) y 2.13 (NET), lo que sugiere la no existencia de autocorrelación de primer orden en los residuos de ambos modelos ( $\mathrm{p}<0.05)$ (Fritts, 1976).

La ecuación de la reconstrucción de la precipitación acumulada de noviembre-mayo para el período completo de calibración (1960-2006) en el sitio NEC es:

$$
\text { Ypp } t=-7.52+176.39 * \mathrm{Xi}
$$


Mientras que la ecuación generada para la reconstrucción de la precipitación acumulada de diciembre-mayo para el período completo (1962-2009) en el sitio NET es:

$$
\text { Yppt }=-21.32+169.72 * \mathrm{Xi}
$$

donde:

Yppt $=$ precipitación acumulada $(\mathrm{mm})$

$\mathrm{Xi}=$ índice de anchura total del anillo de la cronología residual

TABLA 1. Períodos de calibración y verificación y estadísticos de los modelos de regresión lineal entre los registros de precipitación acumulada y las cronologías residuales de los sitios NEC y NET.

\begin{tabular}{|c|c|c|c|c|c|c|c|c|c|}
\hline \multirow{2}{*}{ Sitio } & \multirow[t]{2}{*}{ Prueba } & \multirow{2}{*}{ Período } & \multirow{2}{*}{$r^{2} A d j$} & \multicolumn{2}{|c|}{ Coeficiente (mm) } & \multicolumn{2}{|c|}{ Error estándar (mm) } & \multirow{2}{*}{$\begin{array}{c}\text { Valor } \\
\text { prueba-t }\end{array}$} & \multirow{2}{*}{$\begin{array}{c}\text { Vvalor } \\
\text { prueba- } f\end{array}$} \\
\hline & & & & $B_{0}$ & $B_{1}$ & $B_{0}$ & $B_{1}$ & & \\
\hline \multirow{3}{*}{ NEC } & Calibración & $1960-1983$ & 0.38 & -48.15 & 215.37 & 60.22 & 55.39 & $3.89^{*}$ & $15.12^{*}$ \\
\hline & Verificación & 1984-2006 & 0.73 & 4.78 & 161.97 & 20.56 & 20.75 & $7.80^{*}$ & $60.92^{*}$ \\
\hline & Completo & 1960-2006 & 0.57 & -7.52 & 176.39 & 23.38 & 22.46 & $7.85^{*}$ & $61.68^{*}$ \\
\hline NET & Completo & 1962-2009 & 0.53 & -21.32 & 169.72 & 23.33 & 22.84 & $7.43^{*}$ & $55.20^{*}$ \\
\hline
\end{tabular}

$r^{2}$ Adj: $r^{2}$ ajustada *Significancia $p<0.01$
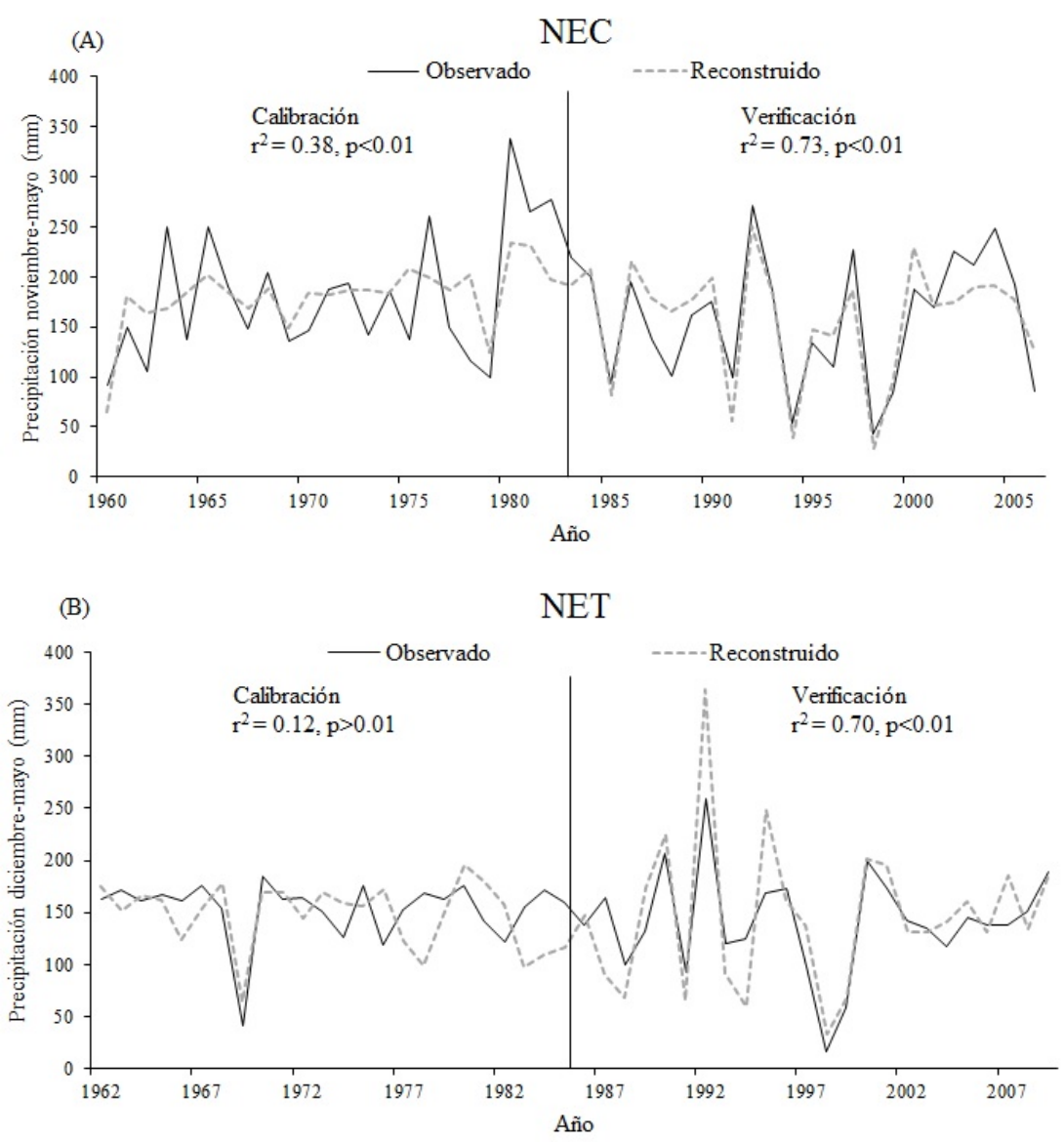

FIGURA 5. Comparación entre los datos observados y reconstruidos de precipitación estacional del NEC (A) y NET (B) en la Faja Volcánica Transmexicana. 
TABLA 2. Estadísticos de verificación entre los datos observados de la precipitación estacional mensual y los reconstruidos para los sitios NEC y NET.

\begin{tabular}{|c|c|c|c|c|c|c|}
\hline Sitio & Período & $\begin{array}{c}\text { Coeficiente de } \\
\text { correlación }\end{array}$ & $\begin{array}{c}\text { Reducción del } \\
\text { error }^{1}\end{array}$ & $\begin{array}{l}\text { Valor prueba } \\
\text { de signos }\end{array}$ & $\begin{array}{c}\text { Valor prueba- } \\
t\end{array}$ & $\begin{array}{c}\text { Primera } \\
\text { diferencia } \\
\text { negativa }^{2}\end{array}$ \\
\hline \multirow{2}{*}{ NEC } & 1960-1983 & $0.64^{*}$ & 0.39 & $8^{*}$ & $2.16^{*}$ & $7^{*}$ \\
\hline & 1984-2006 & $0.86^{*}$ & 0.78 & $4^{*}$ & $3.05^{*}$ & $1^{*}$ \\
\hline \multirow{2}{*}{ NET } & 1962-1985 & $0.40^{*}$ & -0.15 & 12 & 0.99 & 14 \\
\hline & 1986-2009 & $0.84^{*}$ & 0.68 & $6^{*}$ & $2.51^{*}$ & $6 *$ \\
\hline
\end{tabular}

(1): No se dispone de alguna prueba de significancia para esta estadística, pero cualquier resultado positivo indica que la reconstrucción aporta una información paleoclimática útil (Fritts, 1976). (2): Primera diferencia entre los datos observados y los reconstruidos [ $\mathrm{t}$ - (t-1)], la transformación elimina las tendencias que pueden afectar el coeficiente de correlación (Fritts, 1976). *Significativo p<0.05.

Los índices de ancho de anillo explicaron en 57\% y 53\% la variabilidad de los datos instrumentales de la precipitación estacional en los sitios NEC y NET, respectivamente (Fig, 6). La función de respuesta entre el sitio POR y precipitación estacional de diciembre del año previo a mayo del año actual, se descartó del análisis, debido que la varianza explicada por el crecimiento anual no fue significativa y la extensión de la cronología fue muy corta.

La reconstrucción de la precipitación estacional de noviembre-mayo para el período 1940-2016 en NEC indicó una media histórica de $172.75 \mathrm{~mm}$ y una desviación estándar ( $\sigma$ ) de $42.58 \mathrm{~mm}$. En cambio, en NET se registró un promedio histórico de $152.7 \mathrm{~mm}$ y una desviación estándar $(\sigma)$ de $39.87 \mathrm{~mm}$ para la reconstrucción de la precipitación de diciembre-mayo de 1928-2016. Las condiciones de años de sequías y de humedad extremas registradas $( \pm 2 \sigma)$ por los árboles muestreados en los sitios NEC y NET se presentan en la tabla 3 y las figuras 7, 8 y 9. Los años secos y húmedos identificados en este estudio fueron comparados con lo publicado en la literatura para la región de estudio.

\section{Influencia de fenómenos océano-atmósfera en $P$. hartwegii}

Los resultados del análisis de influencia de los índices de ENOS (IEM, INO y IOS) y las cronologías (arstan, estándar y residual) indicaron asociaciones significativas ( $\mathrm{p}$
$<0.05)$ sobre el crecimiento arbóreo del sitio NEC. Sin embargo, estos índices presentaron una influencia nula $(\mathrm{p}$ $>0.05)$ en el crecimiento de los árboles de los sitios NET y POR (Tabla 4). Por otra parte, los tres índices de ENOS influyeron significativamente en las cronologías estándar de NEC. El IEM tuvo una influencia positiva durante los meses de junio-diciembre de 1980-2015 ( $\mathrm{r}=0367, \mathrm{p}<0.05)$ al igual que el INO durante mayo-diciembre de 1951-2015 $(\mathrm{r}=0.265, \mathrm{p}<0.05)$, mientras que el IOS mostró una influencia negativa durante septiembre-enero de 1951-2015 $(\mathrm{r}=-0.322, \mathrm{p}<0.05)$.

En cambio, la ODP ejerció una influencia positiva significativa en la cronología estándar de NEC durante septiembre-noviembre de 1940-2016 $(\mathrm{r}=0.24, \mathrm{p}<0.05)$, mientras que en NET (cronología arstan) y POR (cronología estándar) la influencia fue negativa durante octubre-diciembre de 1950-2014 ( $\mathrm{r}=-0.319, \mathrm{p}<0.05)$ y de marzo de 1960-2016 ( $\mathrm{r}=-0.30, \mathrm{p}<0.05)$, respectivamente (Tabla 4).

La OMA influyó de manera negativa significativa $(\mathrm{p}<0.05)$ en el crecimiento de los árboles en NEC (cronología estándar) durante enero-abril de 1940-2014, sin embargo, sus efectos fueron positivos sobre el crecimiento de los árboles de NET (cronología estándar) y POR (cronología residual) durante mayo-diciembre de 1930$2010(\mathrm{r}=0.234, \mathrm{p}<0.05)$ y mayo-diciembre de 1960-2014 $(\mathrm{r}=0.35, \mathrm{p}<0.05)$, respectivamente (Tabla 4$)$. 

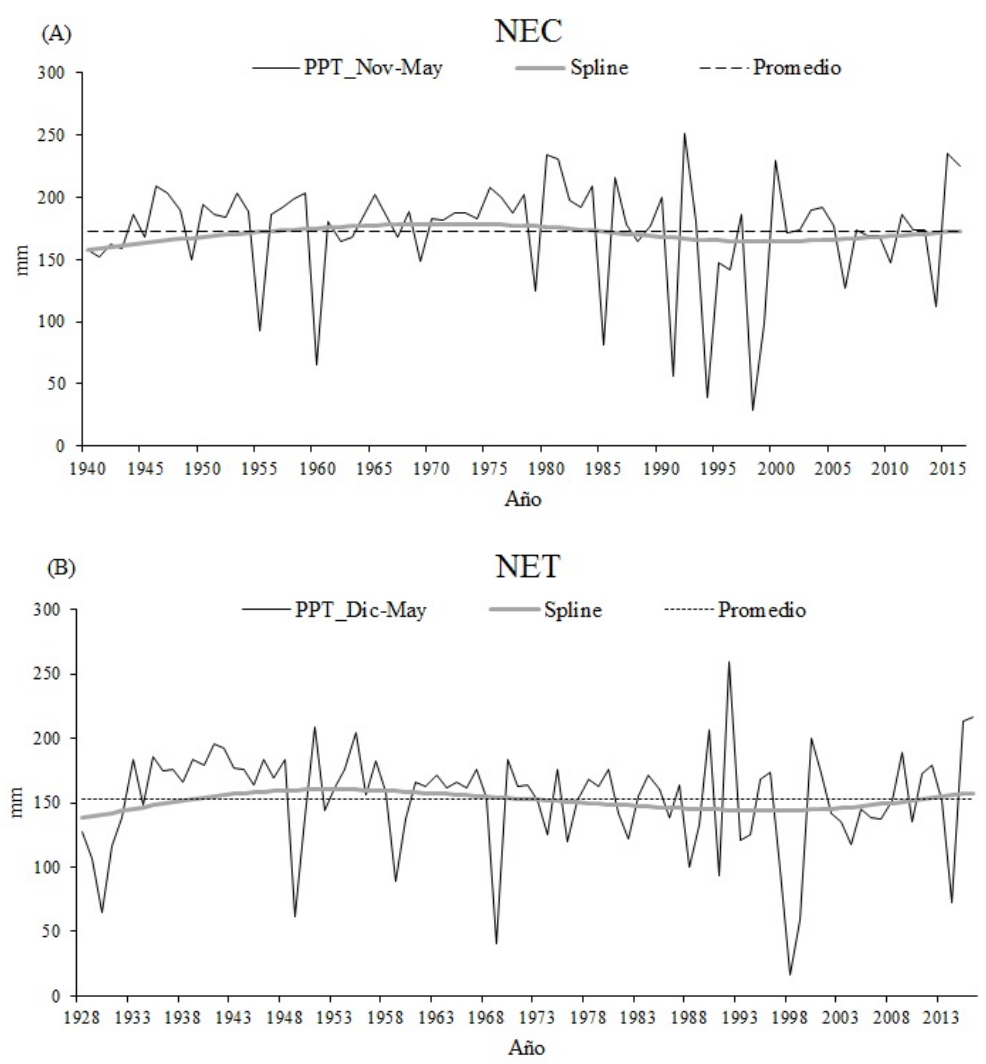

FIGURA 6. Reconstrucción de precipitación estacional noviembre-mayo (1940-2016) en el NEC (A) y diciembre-mayo (1928-2016) en el NET (B).

Spline (curva suavizada). Promedio (promedio histórico de precipitación).

TABLA 3. Años de sequias y humedad extremas registrados en los sitios NEC y NET.

\begin{tabular}{lccccc}
\hline Sitio & Año & $\begin{array}{c}\text { Precipitación estacional } \\
(\mathrm{mm})\end{array}$ & $\begin{array}{c}\text { Media histórica } \\
(\mathrm{mm})\end{array}$ & $\begin{array}{c}\text { Reducción de } \\
\text { precipitación (\%) }\end{array}$ & $\begin{array}{c}\text { Aumento de } \\
\text { precipitación }(\%)^{2}\end{array}$ \\
\hline NEC & 1960 & 64.81 & 172.75 & 62.48 & \\
& 1985 & 81.57 & 172.75 & 52.78 & \\
& 1991 & 55.98 & 172.75 & 67.59 & \\
& 1994 & 39.04 & 172.75 & 77.39 & \\
NET & 1992 & 251.2 & 172.75 & & \\
& 1998 & 28.43 & 172.75 & 83.54 & \\
& 1930 & 64.68 & 152.7 & 57.64 & \\
& 1949 & 61.26 & 152.7 & 59.88 & \\
& 1969 & 41.03 & 152.7 & 73.13 & \\
& 1992 & 259 & 152.7 & & \\
& 1998 & 16.31 & 152.7 & 89.32 & \\
& 1999 & 59.25 & 152.7 & 61.2 & \\
& 2014 & 73 & 152.7 & 52.2 & \\
\hline
\end{tabular}

Reducción!: valor calculado con respecto a la disminución de la precipitación media histórica.

Aumento de precipitación $(\%)^{2}$ : valor calculado con respecto al aumento de la precipitación media histórica. 


\section{PDSI}

\section{Noviembre-Mayo}


Figura 7. Mapas de sequías generados con el Atlas de sequías para México (Stahle et al., 2016). Condiciones de sequías en el centro de México y su coincidencia con los años más extremos de las sequías reconstruidas con anillos de crecimiento de $P$. hartwegii en el Nevado de Colima (NEC).

Los valores -6 (color rojo) y 6 (color azul) en la escala PDSI indican condiciones de sequía extrema y de humedad extrema. La estrella negra en los mapas representa la ubicación del sitio NEC.

\section{DISCUSIÓN}

\section{Calidad de cronologías}

Los valores de correlaciones entre series de 0.585 (NEC), 0.516 (NET) y 0.412 (POR) son mayores al umbral de 0.328 $(\mathrm{p}<0.01)$ establecido por Holmes (1983), lo cual otorga una confiablidad de $99 \%$ al fechado de las muestras. Valores similares, de 0.385 a 0.5 , han sido obtenidos por Carlón et al. (2021) y Astudillo-Sánchez et al. (2017) para P. hartwegii del centro de México.

La sensibilidad media es el parámetro más relevante en la evaluación del potencial dendrocronológico de especies arbóreas (Speer, 2010; Villanueva-Díaz et al., 2016; Gutiérrez-García y Ricker, 2019). Los valores de sensibilidad media de 0.234 (POR), 0.243 (NET) y 0.314 (NEC) obtenidos para $P$. hartwegii son muy similares a lo 
encontrados por Carlón et al. (2021) en el Pico de Tancítaro (0.239) y por Astudillo-Sánchez et al. (2017) en el Monte Tláloc (0.32). Por lo que la poca variación de la sensibilidad media registrada por $P$. hartwegii en sitios de alta montaña del centro de México, estaría indicando que las condiciones ecológicas de esas localidades son similares.
La baja variabilidad de la autocorrelación de primer orden en NEC, NET y POR indica que el crecimiento arbóreo de esos sitios no está influenciado por las condiciones climáticas del año previo (Fritts, 1976; Speer, 2010), lo que resulta apropiado para registrar la variación climática interanual de baja frecuencia de cada sitio.

\section{PDSI}

\section{Diciembre-Mayo}
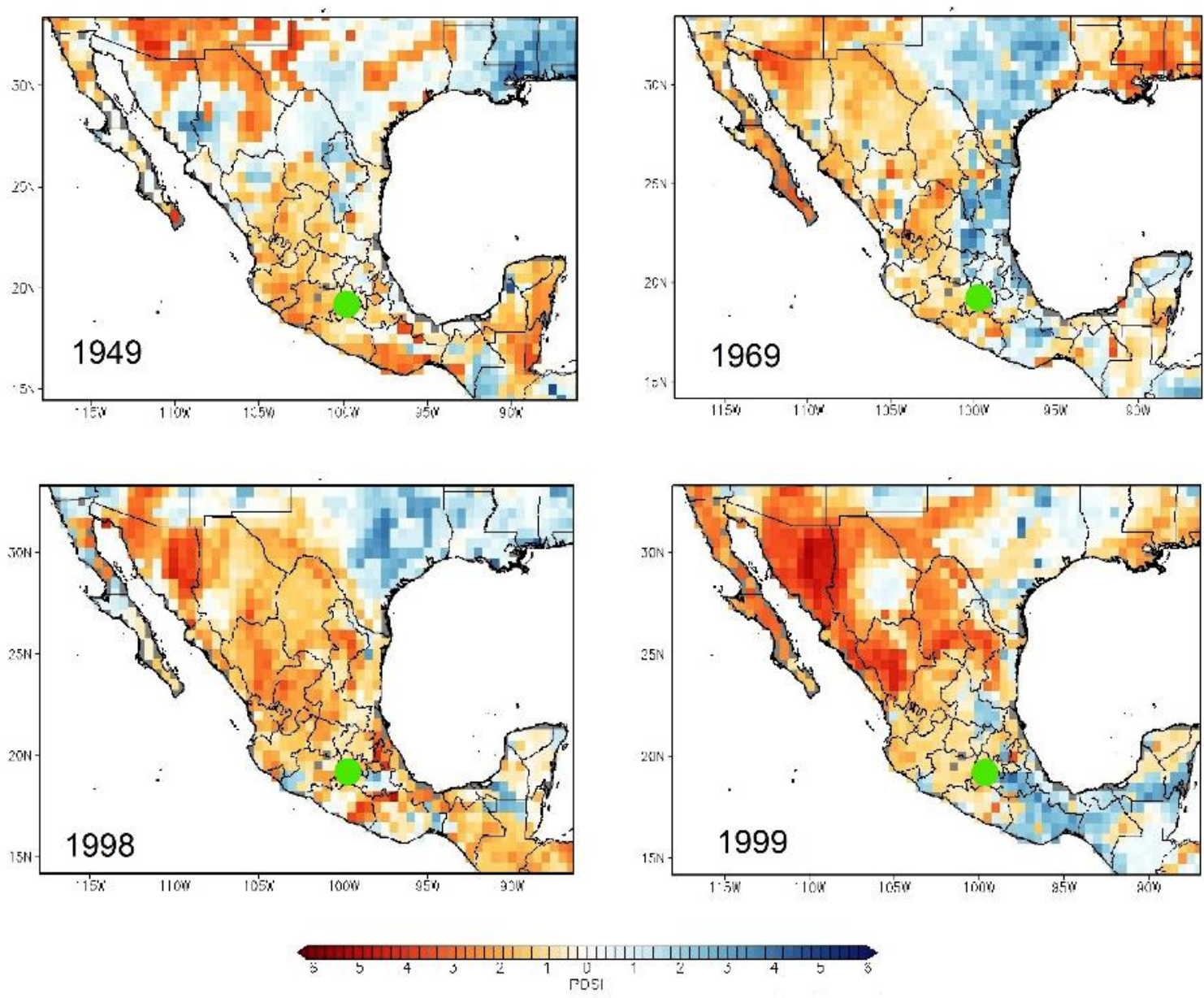

Figura 8. Mapas de sequías generados con el Atlas de sequías para México (Stahle et al., 2016). Condiciones de sequías en el centro de México y su coincidencia con los años más extremos de las sequías reconstruidas con anillos de crecimiento de P. hartwegii en el Nevado de Toluca (NET).

Los valores -6 (color rojo) y 6 (color azul) en la escala PDSI indican condiciones de sequía extrema y de humedad extrema. El círculo verde en los mapas representa la ubicación del sitio NET. 
PDSI Noviembre-Mayo

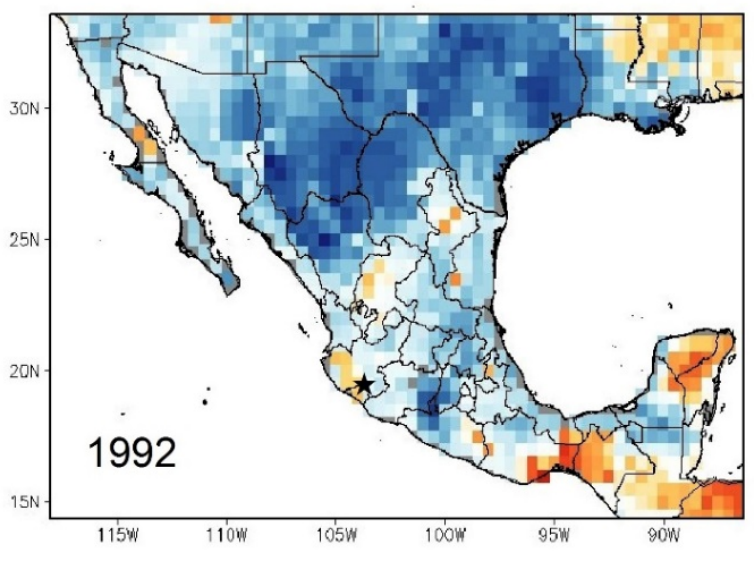

\section{PDSI} Diciembre-Mayo

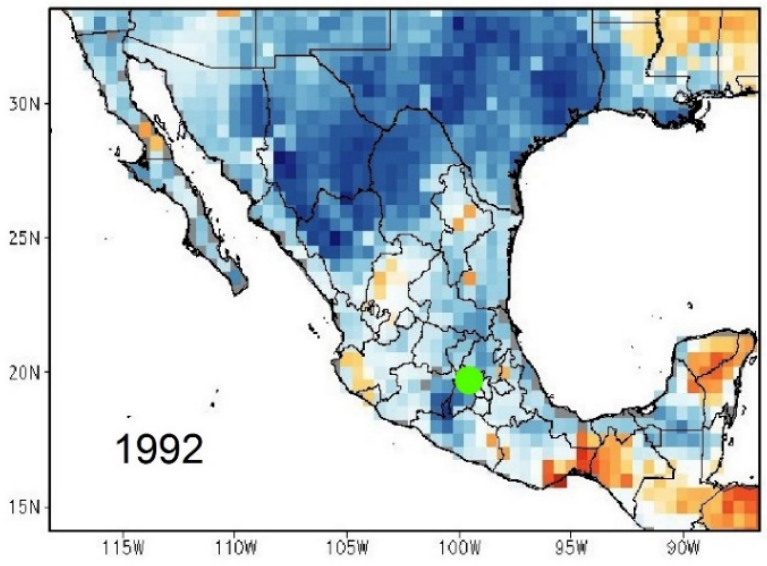

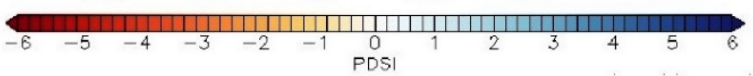

Figura 9. Mapas de humedad generados con el Atlas de Sequías para México (Stahle et al., 2016). Condiciones de humedad en el centro de México y su coincidencia con los años más húmedos extremos reconstruidos con anillos de crecimiento de $P$. hartwegii en el Nevado de Colima (NEC) y Nevado de Toluca (NET).

Los valores -6 (color rojo) y 6 (color azul) en la escala PDSI indican condiciones de sequía extrema y de humedad extrema. La estrella negra representa la ubicación del sitio NEC y el círculo verde representa la ubicación del sitio NET en los mapas.

TABLA 4. Asociación entre las cronologías de P. hartwegii y los índices de fenómenos océano-atmósfera.

\begin{tabular}{|c|c|c|c|c|c|}
\hline \multirow[b]{2}{*}{ Sitio } & \multirow{2}{*}{$\begin{array}{c}\text { Tipo de } \\
\text { cronología }\end{array}$} & \multicolumn{4}{|c|}{ IEM } \\
\hline & & Período estacional & Período (años) & $\begin{array}{c}\text { Coeficiente de correlación de } \\
\text { Pearson }\end{array}$ & Valor $p$ \\
\hline NEC & Estándar & Jun-Dic & $1980-2015$ & 0.367 & $0.029^{*}$ \\
\hline NET & $* *$ & ** & ** & $* *$ & $* *$ \\
\hline \multirow[t]{2}{*}{ POR } & $* *$ & ** & ** & ** & ** \\
\hline & & \multicolumn{4}{|c|}{ INO } \\
\hline NEC & Estándar & May-Dic & $1951-2015$ & 0.265 & $0.043^{*}$ \\
\hline NET & $* *$ & $* *$ & ** & $* *$ & $* *$ \\
\hline \multirow[t]{2}{*}{ POR } & ** & ** & ** & ** & $* *$ \\
\hline & & \multicolumn{4}{|c|}{ IOS } \\
\hline NEC & Estándar & Sep-Ene & 1951-2015 & -0.322 & $0.008^{*}$ \\
\hline NET & ** & ** & $* *$ & $* *$ & ** \\
\hline \multirow[t]{2}{*}{ POR } & $* *$ & ** & ** & $* *$ & $* *$ \\
\hline & \multicolumn{5}{|c|}{ ODP } \\
\hline NEC & Estándar & Sep-Nov & $1940-2016$ & 0.244 & $0.032 *$ \\
\hline NET & Arstan & Oct-Dic & 1950-2014 & -0.319 & $0.010^{*}$ \\
\hline \multirow[t]{2}{*}{ POR } & Estándar & Marzo & $1960-2016$ & -0.298 & $0.024^{*}$ \\
\hline & \multicolumn{5}{|c|}{ OMA } \\
\hline NEC & Estándar & Ene-Abr & $1940-2014$ & -0.341 & $0.003^{*}$ \\
\hline NET & Estándar & May-Dic & 1930-2010 & 0.234 & $0.036^{*}$ \\
\hline POR & Residual & May-Dic & 1960-2014 & 0.347 & $0.009^{*}$ \\
\hline
\end{tabular}


La variación del primer componente principal registrada fue de 40.33 (NEC), 37.64 (NET) y 21.48\% (POR). Estos resultados reflejan una señal común de crecimiento arbóreo, con valores que oscilan entre 0\% y 100\% (Briffa y Jones, 1990; Speer, 2010). Por lo tanto, entre mayor sea esta variación, mayor será la señal de crecimiento registrada por los árboles.

La SEP confiable $(\geq 0.85)$ se obtuvo a las nueve muestras (período 1940-2016) en NEC, 12 muestras (período 1928-2016) en NET, sin embargo, este valor confiable no se alcanzó en POR (SEP $=0.80,18$ muestras) período 1960-2016, probablemente debido a otros factores ajenos, que pudieron influir en el crecimiento de esos árboles.

\section{Respuesta climática}

La respuesta negativa significativa de $P$. hartwegii con la temperatura máxima de marzo-mayo $(p<0.05)$ en NEC es similar a la registrada durante enero-mayo por GutiérrezGarcía y Ricker (2019) en el noreste de México, aunque difiriere con lo obtenido para NET y POR, donde un incremento en la temperatura máxima durante septiembrefebrero y junio-octubre, afecta negativamente $(\mathrm{p}<0.05)$ el período de culminación de crecimiento de la especie en México (Biondi et al., 2005), resultados que concuerdan con lo señalado en estudios previos que encontraron que un incremento en la temperatura máxima tiene efecto negativo en el crecimiento radial de $P$. hartwegii del Pico de Tancítaro, Michoacán (Carlón et al., 2021). Contrariamente a estos resultados, estudios realizados en el Monte Tláloc (Valle de México) para $P$. hartwegii, indican que el aumento de la temperatura máxima favorece el crecimiento radial de esta especie (Astudillo-Sánchez et al., 2017; 2019). Estos resultados contradictorios pueden explicarse por las condiciones microambientales locales de cada sitio donde la especie crece, indicando así, que el efecto que tiene esta variable en la reactivación del cambium vascular de $P$. hartwegii se debe a las condiciones ambientales dominantes durante la primavera-verano y a la dinámica forestal (reclutamiento, competencia) de los árboles en cada sitio
(Villanueva-Díaz et al., 2016; Astudillo-Sánchez et al., 2019; Gutiérrez-García y Ricker, 2019).

A su vez, las temperaturas medias y mínimas solamente influyeron de manera negativa $(p<0.05)$ en el crecimiento radial de los árboles muestreados en el POR durante el verano y principios del otoño. Por lo que el aumento de estas variables durante ese período propicia que el crecimiento radial se vea disminuido. Resultados similares evidencian el efecto negativo del aumento de la temperatura promedio anual en el crecimiento radial de Juniperus monticola en el Monte Tláloc (Villanueva-Díaz et al., 2016). Sin embargo, Carlón et al. (2021) difieren con lo anterior, debido a que el aumento de la temperatura mínima durante abril-junio favorece el crecimiento radial de $P$. hartwegii del Pico de Tancítaro. Lo que estaría indicando una capacidad heterogénea de respuesta ambiental de la especie en sitios con condiciones ecológicas similares.

Según estudios recientes en especies de alta montaña, las condiciones ecológicas de los micrositios influyen mucho en la respuesta ambiental de esas especies (Villanueva-Díaz et al., 2016; Astudillo-Sánchez et al., 2019; Carlón et al., 2021; Correa-Díaz, Romero-Sánchez y Villanueva-Díaz, 2021). Por lo tanto, a pesar de que las condiciones ambientales son similares, la respuesta fisiológica en el crecimiento radial de $P$. hartwegii varía acorde con las condiciones microambientales presentes en el sitio donde se desarrolla.

Del mismo modo, la relación entre la precipitación de invierno-primavera y el crecimiento radial de los árboles en los sitios de estudio se atribuye a la cantidad de precipitación recibida en el período frío del año; esta precipitación al ser de baja intensidad queda almacenada en los primeros horizontes del suelo, para después ser aprovechada al inicio de la estación de crecimiento; situación que es favorecida por la presencia de temperaturas estables en el mismo período.

Estas condiciones de precipitación durante los períodos señalados se asocian con un descenso en la temperatura de noviembre a febrero, manteniéndose estables durante marzo a mayo, que es el inicio del período vegetativo de crecimiento de $P$. hartwegii en México y el cual 
culmina en octubre-noviembre (Biondi et al., 2005). Por lo que un aumento en la temperatura durante ese lapso incrementaría la evapotranspiración y disminuiría la humedad disponible del suelo, limitando el crecimiento radial (Dawadi, Liang, Tian, Devkota y Yao, 2013; Thapa, Shah, Gaire y Bhuju, 2015). En cambio, un año con alta precipitación estacional favorecerá el crecimiento por su influencia en la disponibilidad de humedad del suelo, compensando así la pérdida hídrica por procesos de evapotranspiración (Zhou, Fei, Sherry y Luo, 2012).

Estudios realizados en la región suroeste de los Estados Unidos y en el norte y centro de México demuestran que el crecimiento radial anual de las coníferas depende directamente de la precipitación estacional invierno-primavera (Villanueva-Díaz et al., 2015; Stahle et al., 2016; Gutiérrez-García y Ricker, 2019; Carlón et al., 2021); por lo que un déficit hídrico durante el inviernoprimavera suele influir directamente en el crecimiento radial anual de esas especies.

Los eventos de sequías extraordinarias registrados en 1930, 1949, 1955, 1959, 1960, 1991, 1998 y 1999 coinciden con los mencionados por Cerano-Paredes et al. (2013), Stahle et al. (2016) y Villanueva-Díaz et al. (2015; 2018b) para la Faja Volcánica Transmexicana, donde 1998 fue el año más seco registrado a nivel nacional. Los años secos de 1998 y 1999 se catalogan entre los más secos de las últimas siete décadas para el norte y centro de México, incidiendo en 60\% del país (Domínguez, 2016). Simultáneamente, los años secos de 1955 y 1998-1999 coinciden con la ciclicidad de sequías registradas para América del Norte de cada 50 años a 60 años (Stahle et al., 2016).

El único evento (año) de alta humedad extrema registrado en este estudio por los árboles de los sitios NEC y NET corresponde a 1992. Acontecimiento que tuvo una magnitud de afectación de escala nacional, derivado de un episodio intenso de ENOS (1991-1992), que se extendió desde el sur de Estados Unidos hasta el centro de México, afectando 70\% del país (Stahle et al., 2016; Bravo, Azpra, Rodríguez and Rodríguez, 2018).

\section{Influencia de fenómenos océano-atmósfera}

Pese a su alta periodicidad de marcada influencia en México (Pompa-García y Némiga, 2015; Villanueva-Díaz et al., 2015; Aquino-Ramírez et al., 2019). ENOS únicamente influyó de manera significativa sobre el crecimiento radial de los árboles de NEC durante los meses de juniodiciembre de 1980-2015 (IEM), mayo-diciembre de19512015 (INO) y septiembre-enero de 1951-2015 (IOS). Presentando un efecto positivo en el crecimiento radial de $P$. hartwegii con los índices IEM e INO, y un efecto negativo con el índice IOS.

Estudios efectuados por Gutiérrez-García y Ricker (2019) y Carlón et al. (2021) encontraron una influencia positiva significativa de ENOS (temperatura superficial del mar, región 4) y el IEM durante los meses de junio y de marzo-mayo sobre el crecimiento de $P$. hartwegii del noreste y occidente de México, respectivamente. Resultados que son similares a los obtenidos para los índices IEM e INO en NEC. Lo cual indica que las condiciones océanoatmosféricas de ENOS (IEM e INO) de 1951-2010 (Stahle et al., 2016; Bravo et al., 2018) favorecieron el crecimiento de $P$. hartwegii en el noreste y occidente de México, al incrementar la cantidad de precipitación recibida en esos sitios, la cual quedó almacenada en el suelo, para posteriormente ser utilizada durante la etapa de reactivación del crecimiento radial de la especie durante marzo-mayo (Biondi et al., 2005).

Astudillo-Sánchez et al. (2017) y Carlón et al. (2021) observaron una respuesta negativa de ENOS (IOS) en el crecimiento radial de $P$. hartwegii del Monte Tláloc (Valle de México) y el Pico de Tancítaro durante los meses de enerodiciembre y noviembre-diciembre del año previo del crecimiento radial, respectivamente. Estos resultados son similares a lo registrado por los árboles del NEC durante septiembre del año previo a enero del año actual del crecimiento radial.

Este contraste observado de los resultados obtenidos entre índices de ENOS (MEI e IOS) y los períodos de respuesta significativos en el crecimiento radial de $P$. 
hartwegii de los sitios NEC y Pico de Tancítaro (Carlón et al., 2021) pudiera deberse a las variables oceánicas y atmosféricas empleadas en la elaboración de los índices ENOS, de los cuales, IEM es considerado como el más robusto y confiable (Bravo et al., 2018) y es uno de los más empleado en la generación de estudios dendrocronológicos en México (Pompa-García y Némiga, 2015; VillanuevaDíaz et al., 2018b; Aquino-Ramírez et al., 2019; Carlón et al., 2021).

A pesar de que ENOS suele presentar una influencia significativa marcada en el crecimiento radial de muchas especies de coníferas mexicanas (Astudillo-Sánchez et al., 2017; González-Cásares, Pompa-García y Camarero, 2017; Martínez-Sifuentes et al., 2020; Carlón et al., 2021) su afectación en los sitios NET y POR fue prácticamente nula $(p<0.05)$. Estos resultados de muy baja influencia en el crecimiento radial son similares a lo observado en $P$. hartwegii por Manzanilla-Quiñones et al (2020) para el índice ENOS (temperatura superficial del mar, región 3) en el Nevado de Toluca y Pico de Orizaba. Lo cual indica que ENOS no tuvo influencia significativa durante el crecimiento radial de $P$. hartwegii de esos sitios. Estudios similares de nula afectación de ENOS (IEM e IOS) sobre el crecimiento radial de coníferas mexicanas han sido llevados a cabo por Villanueva-Díaz et al. (2018b) para $P$. oocarpa del Bosque la Primavera, en Jalisco y por GonzálezCásares et al. (2017) para $P$. durangensis, en Chihuahua. Lo que sugiere que ENOS no siempre tiene un efecto significativo en el crecimiento radial de las coníferas de México.

El efecto del ODP en el crecimiento fue significativo $(\mathrm{p}<0.05)$ y contrastante; presentando una respuesta positiva $(r=0.244)$ en el crecimiento radial de los árboles situados en el sitio NEC durante los meses de septiembrenoviembre y una respuesta negativa durante los meses de octubre-diciembre $(r=-0.319)$ en NET y en marzo $(r=-$ 0.298 ) en POR. La respuesta positiva obtenida es similar a la observada por Stahle et al. (2012) en Taxodium mucronatum Ten. para el occidente mexicano y por Gutiérrez-García y Ricker (2019), Manzanilla-Quiñones et al. (2020) y Carlón et al. (2021) en P. hartwegii del noreste, Nevado de Colima y Pico de Tancítaro, respectivamente. Lo que indica que la influencia de ODP repercute directamente durante la etapa de crecimiento de la especie y el impacto del fenómeno suele presentarse con mayor intensidad en el norte y occidente de México (Biondi et al., 2001; Álvarez-Olguín y Escalante-Sandoval, 2017; González-Cásares et al., 2017; Gutiérrez-García y Ricker, 2019; Manzanilla-Quiñones et al., 2020; Carlón et al., 2021).

La influencia de OMA mostró un efecto significativo $(p<0.05)$ durante el período enero-abril en el sitio NEC ( $r$ $=-0.341)$ y durante mayo- diciembre en NET $(r=0.234) \mathrm{y}$ POR ( $r=0.347)$. La influencia del fenómeno en las altas montañas de México ha sido escasamente analizada. Sin embargo, se han documentado efectos significativos de OMA en el crecimiento radial de las coníferas del norte de México y en la Península de Yucatán (Méndez y Magaña, 2010; Meko et al., 2013; Villanueva-Díaz et al., 2018a). No obstante, para el centro-occidente del país; ManzanillaQuiñones et al. (2020) y Carlón et al. (2021) observaron una nula influencia y respuesta negativa significativa del fenómeno durante noviembre-diciembre en el crecimiento radial de $P$. hartwegii, respectivamente. Estos últimos, difieren con los resultados obtenidos en este estudio, situación que parece indicar que el efecto de este fenómeno también puede ser modulado por las condiciones locales donde se desarrolla el arbolado, al favorecer o restringir su crecimiento.

Acorde con los resultados obtenidos y a la pregunta de investigación planteada al inicio del estudio; la respuesta climática e influencia de fenómenos circulatorios océanoatmosféricos en el crecimiento radial anual de $P$. hartwegii fue muy variada, incluso entre sitios con condiciones ecológicas análogas, lo que indica, que la influencia de estos fenómenos puede ser modulada por las condiciones ambientales locales donde se desarrolla la especie, favoreciendo su crecimiento y restringiéndolo en otros sitios; excepto en años en los que estos fenómenos han sido de alta intensidad. 


\section{CONCLUSIONES}

Las condiciones ecológicas similares de los sitios de alta montaña donde crece $P$. hartwegii estarían indicando que la especie posee una capacidad heterogénea de respuesta fisiológica a factores climáticos y océano-atmosféricos.

Las series dendrocronológicas desarrolladas permitieron reconstruir y analizar la variabilidad interanual de la precipitación estacional, con las cuales se identificaron y asociaron los principales años de sequías y de humedad extrema calificadas como extraordinarias y de escala nacional.

La influencia de ENOS únicamente fue significativa sobre el crecimiento radial de $P$. hartwegii del sitio NEC, mientras que la afectación de la ODP y OMA fueron significativos en los tres sitios estudiados; sin embargo, este efecto entre sitios se ve modificado por las condiciones ambientales locales donde se desarrolla la especie.

\section{REFERENCIAS}

Acosta-Hernández, A. C., Pompa-García, M., \& Camarero, J. J. (2017). An update review of dendrochronological investigations in Mexico, a megadiverse country with a high potential for tree-ring sciences. Forests, 8(5), 160. doi: doi: 10.3390/f8050160.

Álvarez-Olguín, G., \& Escalante-Sandoval, C. (2017). Modes of variability of annual and seasonal rainfall in Mexico. Journal of the American Water Resources Association, 53(1), 144-157. doi: 10.1111/1752-1688.12488

Aquino-Ramírez, M., Velázquez-Martínez, A., Villanueva-Díaz, J., Hervent-Zamora, H. L., Gómez-Guerrero, A., Reyes-Hernández, V. J., \& Ramírez-Valverde, G. (2019). Respuesta climática de Abies guatemalensis Rehder en Ixtlán de Juárez, Oaxaca, México. Madera y Bosques, 25(2), e2521773. doi: $10.21829 /$ myb.2019.2521773

Astudillo-Sánchez, C. C., Villanueva-Díaz, J., Endara-Agramont, A. R., Nava-Bernal, G. E., \&Gómez-Albores, M. A. (2017). Climatic variability at the treeline of Monte Tláloc, Mexico: a dendrochronological approach. Trees, 31, 441-453. doi: 10.1007/s00468-016-1460-z

Astudillo-Sánchez, C. C., Fowler, M. S., Villanueva-Díaz, J., EndaraAgramont, A. R., Soria-Díaz, L. (2019). Recruitment and facilitation in Pinus hartwegii, a Mexican alpine treeline ecotone, with potential responses to climate warming. Trees, 1-14. doi: 10.1007/s00468-019-01844-3
Biondi, F., Gershunov, A., \& Cayan, D. R. (2001). North Pacific Decadal climate variability since 1661. Journal of Climate, 14, 5-10. doi: 10.1175/1520-0442(2001)014<0005:NPDCVS>2.0.CO;2

Biondi, F., \& Waikul, K. (2004). DendroClim2002: a C++ program for statistical calibration of climate signals in tree-ring chronologies. Computers and Geosciences, 30, 303-311. doi: 10.1016/j.cageo.2003.11.004

Biondi, F., Hartsough, P. C., \& Galindo-Estrada, I. (2005). Daily weather and tree growth at tropical treeline of North America. Artic, Antarctic and Alpine Research, 37, 6-24. doi: 10.1657/15230430(2005)037[0016:dwatga]2.0.co;2.

Bravo, C. J. L., Azpra, R. E., Rodríguez, G. F. J., \& Rodríguez, L. O. (2018). Effects of ENSO on precipitation in Mexico City. Investigaciones Geográficas, 97, 1-12. doi: 10.14350/rig.59679

Briffa, K. R., \& Jones, P. D. (1990). Basic chronology statistics and assessment. En E. R. Cook, \& L. A. Kairiukstis (Eds.). Methods of dendrochronology: applications in the environmental sciences (pp. 137-152). Boston. USA: Kluwer Academic Publishers.

Campos-Aranda, D. F. (1998). Procesos del Ciclo Hidrológico. Capitulo: Regresión y correlación lineal (pp. 1-12). San Luis Potosí, México: Editorial Universitaria Potosina.

Carlón, A. T., Villanueva, D. J., Soto, C. G., Mendoza, M. E., \& Macías, J. L. (2021). Tree rings as indicators of climatic variation in the Trans-Mexican Volcanic Belt, central Mexico. Ecological Indicators, (120), 1-12. doi: 10.1016/j.ecolind.2020.106920

Centro de Investigación Científica y de Educación Superior de Ensenada [Cicese] (s/f). Base de datos climatologica nacional (Sistema Clicom). Recuperado de http://clicom-mex.cicese.mx/)

Cerano-Paredes, J., Villanueva-Díaz, J., Vázquez-Selem, L., CervantesMartínez, R., Magaña-Rueda, V. O., Constante-García, V., Esquivel-Arriaga, G., \& Valdez-Cepeda, D. (2019). Climatic influence on fire regime (1700 to 2008) in the Nazas watershed, Durango, Mexico. Fire Ecology, 15(9), 1-14. doi: 10.1186/s42408018-0020-x

Cook, E. R., \& Holmes, R. L. (1986). User's manual for program Arstan. En R. L. Holmes, R. K. Adams, E. D. Fritts (Eds.), Tree-ring chronologies of western North America: California, eastern Oregon and northern Great Basin (pp. 50-65). Tucson, Arizona, USA: Tucson, Laboratory of Tree-Ring Research, University of Arizona.

Cook, E. (1987). The decomposition of tree-ring series for environmental studies. Tree Ring Bulletin, 47, 37-59.

Cook, E. R., Seager, R., Cane, M. A., \& Stahle, D. W. (2007). North American drought: reconstruction, causes and consequences. Earth Science Review, 81(1-2), 93-134. doi: 10.1016/j.earscire.2006.12.002 
Correa-Díaz, A., Romero-Sánchez, M. R., \& Villanueva-Díaz, J. (2021). The greening effect characterized by the Normalize Difference Vegetation Index was not coupled with phenological trends and tree growth rates in eight protected mountains of central Mexico. Forest Ecology and Management, 496, (2021), 119402. doi: 10.1016/j.foreco.2021.119402

Dawadi, B., Liang, E., Tian, L., Devkota, L. P., \& Yao, T. (2013). Premonsoon precipitation signal in tree rings of timberline Betula utilis in the central Himalayas. Quaternary International, 283, 72-77. doi: 10.1016/j.quaint.2012.05.039

Domínguez, J. (2016). Revisión histórica de las sequías en México: de la explicación divina a la incorporación de la ciencia. Tecnología y Ciencias del Agua, 7(5), 77-93.

Farjon, A., Pérez de la Rosa, J., \& Styles, T. B. (1997). Guía de campo de los pinos de México y América Central. Royal Botanic Gardens, Kew University of Oxford.

Fritts, H. C. (1976). Tree rings and climate. Academic Press, London, $567 \mathrm{p}$.

García, E. (1998). Climas. (Clasificación de Köppen, modificado por García). Escala 1:1000000. Comisión Nacional para el Conocimiento y Uso de la Biodiversidad (Conabio). Archivo Shapefile. México, D. F.

González-Cásares, M., Pompa-García, M., \& Camarero, J. J. (2017). Differences in Climate-growth relationship indicate diverse drought tolerances among five pin species coexisting in Northwestern Mexico. Trees, 31, 531-544. doi: 10.1007/s00468016-1488-0

Gutiérrez-García, G., y Ricker, M. (2019). Influencia del clima en el crecimiento radial en cuatro especies de coníferas en la sierra de San Antonio Peña Nevada (Nuevo León, México). Revista Mexicana de Biodiversidad, 90(2019), e902676

Holmes, R. L. (1983). Computer-assisted quality control in tree-ring dating and measurement. Tree Ring Bulletin, 43, 69-78.

Holmes, R. L. (1994). Dendrocbronology Program Library (DPL). Laboratory of Tree-Ring Research, University. Recuperado de http://www.ltrr.arizona.edu/software.html.

Instituto Nacional de Estadística y Geografía (Inegi) (2014). Conjunto de datos vectoriales edafológicos. Escala 1:250 000. Serie II. Archivo Shapefile. México, D.F., México.

Instituto Nacional de Estadística y Geografía (Inegi) (2016). Uso del suelo y vegetación. Escala 1:250 000. Serie VI (capa unión). Archivo Shapefile. México, D.F., México.

Mastrandrea, T. E. Billir, M.Chatterjee, K. L. Ebi, Y. O. Estrada, R. C. Genova, B. Girma, E. S. Kissel, A. N. Levy, S. MacCracken, P. R.
Körner, C. (1998). A re-assessment of high elevation treeline positions and their explanation. Oecologia, 115(4), 445-459. doi: $10.1007 / \mathrm{s} 004420050540$

Kohler, T., Wehrli, A. \& Jurek, M., (Eds). (2014). Mountains and climate change: A global concern. Sustainable Mountain Development Series. Bern, Switzerland, Centre for Development and Environment (CDE), Swiss Agency for Development and Cooperation (SDC) and Geographica Bernensia.

Manzanilla-Quiñones, U., Aguirre-Calderón, O. A., Jiménez-Pérez, J., Treviño-Garza, E. J., \& Yerena-Yamallel, J. I. (2019). Distribución actual y futura del bosque subalpino de Pinus hartwegii Lindl en el Eje Neovolcánico Transversal. Madera y Bosques, 25 (2), e2521804. doi: 10.21829/myb.2019.2521804

Manzanilla-Quiñones, U., Aguirre-Calderón, O. A., Jiménez-Pérez, J., \& Villanueva-Díaz, J. (2020). Sensibilidad climática en anchuras de anillos de crecimiento de Pinus hartwegii: una especie alpina mexicana con potencial dendroclimático. Revista Mexicana de Biodiversidad, 91(2020), e913117. doi: 10.22201/ib.20078706e.2020.91.3117

Martínez-Sifuentes, A. R., Villanueva-Díaz, J., \& Estrada-Ávalos, J. (2020). Runnoff reconstruction and Climatic influence with tree rings, in the Mayo River basin, Sonora, Mexico. Iforest, 13, 98-106. doi: 10.3832/ifor3190-013

Meko, D. M., Touchan, R., Villanueva-Díaz, J., Griffin, D., Woodhouse, C. A., Castro, C. L., Carrillo, C., \& Leavitt, W. (2013). Sierra San Pedro Mártir, Baja California, cool-season precipitation reconstructed from earlywood width of Abies concolor tree rings. Journal of Geophysical Research Biogeosciences, 118, 1660-1673. doi: 10.1002/2013JG00208

Méndez, M. y Magaña, V. (2010). Regional aspects of prolonged meteorological droughts over Mexico and Central America. Journal of Climate, 23, 1175-1188. doi: 10.1175/2009jcli3080.1

NOAA Physical Sciences Laboratory [PSL]. https://psl.noaa.gov/enso/data.html?fbclid=IwAR11TnhBVjku zgqCT_BIctK6-

W7m6Dd3YePYmFmZoZXD1sNDUBOiJmBuoKc)

NOAA Physical Sciences Laboratory [PSL] https://www.esrl.noaa.gov/psd/data/climateindices/list/

Panel Intergubernamental de Expertos sobre Cambio Climático [IPCC] (2014). Resumen para responsables de políticas. Contribución del Grupo de trabajo II al Quinto Informe de Evaluación del Panel Intergubernamental de Expertos sobre el Cambio Climático. En C. B. Field, V. R. Barros, D. J. Dokken, K. J. Mach, M. D. Mastrandrea., \& L. L. White (Eds.), Cambio climático 2014. Impactos, adaptación y vulnerabilidad. Ginebra, Suiza: IPCC. 
Pompa-García, M., Dávalos-Sotelo, R., Rodríguez-Téllez, E., AguirreCalderón, O. A., \&Treviño-Garza, E. J. (2014). Sensibilidad climática de tres versiones dendrocronológicas para una conífera mexicana. Madera y Bosques, 20(3), 139-151. doi: $10.21829 /$ myb.2014.203158

Pompa-García, M., \& Némiga, X. A. (2015). ENSO index teleconnection with seasonal precipitation in a temperate ecosystem of northern Mexico. Atmósfera, 28(1), 43-50. doi: 10.1016/S01876236(15)72158-2

R Core Team (2019). R: A language and environment for statistical computing. R Foundation for Statistical Computing. Vienna, Austria. Recuperado de htpp://www.r-project.org

Sáenz-Romero, C., Rehfeldt, G. E., Crookston, N. L., Pierre, D., StAmant, R., Beaulieu, J., \& Richardson, B. (2010). Contemporary and projected Spline Climate surfaces for Mexico and their use in understanding Climate-plant relationships. Climatic Change, 102(34), 595-623. doi: 10.1007/s10584-009-9753-5

Schofield, M. R., Barker, R. J., Gelman, A., Cook, E. R., \& Briffa, K. R. (2016). A model based approach to climate reconstruction using tree-ring data. Journal of the American Statistical Association, 111(513), 93-106. doi: 10.1080/01621459.2015.1110524

Schulz, E. F. (1976). Problems in applied hydrology. En E. F Schulz (Ed.), Elementary statistical properties (pp. 31-73). Fort Collins, Colorado, USA: Water Resources Publications.

Speer, J. H. (2010). Fundamentals of tree ring research. Tucson, Arizona, USA: University of Arizona Press.

Stahle, D. W., Burnette, D. J., Díaz, J. V., Heim, R. R., Fye, J. F. K., Paredes, J. C., Soto, R. A., \& Cleaveland, M. K. (2012). Pacific and Atlantic influences on Mesoamerican climate over the past millennium. Climate Dynamics, 39(6), 1431-1446. doi: $10.1007 / \mathrm{s} 00382-011-1205-\mathrm{z}$

Stahle, D. W., Cook, E. R., Burnette, D., Villanueva, D. J., Cerano, P. J., Burns, J., Griffin, D., \& Howard, J.M. (2016). The Mexican drought atlas: Tree-ring reconstructions of the soil moisture balance during the late pre-Hispanic, colonial, and modern eras. Quaternary Science Reviews, 149, 34-60. doi: 10.1016/j.quascirev.2016.06.018

Stahle, D. W., Cook, E. R., Burnette, D. J., Torbenson, M. C. A., Howard, I. M., Griffin, D., Villanueva, D. J., Cook, B. I., Williams, A. P., Watson, E., Sauchyn, D. J., Pederson, N., Woodhouse, C. A., Pederson, G. T., Meko, D., Coulthard, B., \& Crawford, C. J. (2020). Dynamics, variability and change in seasonal precipitation reconstructed for North American. American Meteorological Society Journal of Climate, 33, 3173-3195. doi: 10.1175/JCLI-D-19-0270.1
Sun, C., Liu, Y., Song, H., Mei, R., Payomrat, P., Wang, L., \& Liu, R. (2018). Tree-ring-based precipitation reconstruction in the source region of Weihe River, northwest China since AD 1810. International Journal of Climatology, 38(8), 3421-3431. doi: 10.1002/joc. 5514

Thapa, U. K., Shah, S. K., Gaire, N. P., \& Bhuju, D. R. (2015). Spring temperatures in the far-western Nepal Himalaya since AD 1640 reconstructed from Picea smithiana tree-ring widths. Climate dynamics, 45(7-8), 2069-2081. doi: 10.1007/s00382-014-2457-1

Villanueva-Díaz, J., Cerano-Paredes, J., Vázquez-Selem, L., Stahle, D. W., Fulé, P. Z., Yocom, L. L., Francv-Ramos, O., \& Ruiz-Corral, J. A. (2015). Red dendrocronológica del pino de altura (Pinus hartwegii Lindl.) para estudios dendroclimáticos en el noreste y centro de México. Investigaciones Geográficas, 86, 5-14. doi: 10.14350/rig. 42003

Villanueva-Díaz, J., Vázquez-Selem, L., Gómez-Guerrero, A., CeranoParedes, J., Aguirre-González, N. A., \& Franco-Ramos, O. (2016). Potencial dendrocronológico de Juniperus monticola Martínez en el Monte Tláloc, México. Revista Fitotecnia Mexicana, 3(2), 175-185.

Villanueva-Díaz, J., González-Elizondo, M., Cerano-Paredes, J., Estrada-Ávalos, J., Martínez-Sifuentes, A. R., \& Rosales-Mata, S. (2018a). Red dendrocronológica de pino triste (Pinus lumboltziii B. L. Rob. \& Fernald) en la Sierra Madre Occidental para reconstrucción de lluvia estacional. Madera y Bosques, 24(2), e2421530. doi: 10.21829/myb.2018.2421530

Villanueva-Díaz, J., Rubio-Camacho, A., Chávez-Durán, A. A., ZavalaAguirre, J. J., Cerano-Paredes, J., \& Martínez-Sifuentes, A. R. (2018b). Respuesta climática de Pinus oocarpa Schiede Ex Schetol en el Bosque La Primavera, Jalisco. Madera y Bosques, 24(1), e2411464. doi: 10.21829/myb.2018.241464

Zhou, X. H., Fei, S. F., Sherry, R., \& Luo, Y. Q. (2012). Root biomass dynamics under experimental warming and doubled precipitation in a tallgrass prairie. Ecosystems, 15, 542-554. doi: 10.1007/s10021012-9525-3

Manuscrito recibido el 10 de octubre de 2020

Aceptado el 24 de junio de 2021

Publicado el 30 de diciembre de 2021 
Este documento se debe citar como:

Manzanilla-Quiñones, U., Aguirre-Calderón, O. A., Villanueva-Díaz, J., Martínez-Sifuentes, A. R., \& DelgadoValerio, P. (2021). Anillos de crecimiento de Pinus hartwegii como indicadores de fluctuaciones climáticas y de la influencia de fenómenos océano-atmósfera en la Faja Volcánica Transmexicana. Madera y Bosques, 27(3), e2732276. doi: 10.21829/myb.2021.2732276

Madera y Bosques por Instituto de Ecología, A.C. se distribuye bajo una Licencia Creative Commons Atribución-NoComercialCompartirlgual 4.0 Internacional. 